\title{
Depresión, ansiedad y autoeficacia para bajar de peso en mujeres en tratamiento nutricional
}

\section{Depression, anxiety and self-efficacy to lose weight in women in a nutritional treatment}

\author{
Cecilia Silva, Natalia Fernández y Natalia Rodríguez ${ }^{1}$ \\ Citación: Silva, C., Fernández, N. y Rodríguez, N. (2019). Depresión, ansiedad y autoeficacia para \\ bajar de peso en mujeres en tratamiento nutricional. Psicología y Salud, 29(1), 41-49.
}

\section{RESUMEN}

\begin{abstract}
Con la finalidad de explorar la relación entre depresión, ansiedad, autoeficacia para bajar de peso y cambio de peso corporal en mujeres con sobrepeso y obesidad sometidas a tratamiento nutricional, se llevó a cabo un estudio trasversal de tipo correlacional, donde se aplicaron el IDARE, el BDI-II y la Escala de Autoeficacia a 122 pacientes de dos clínicas de medicina familiar de Ciudad de México. Los resultados muestran que entre las personas obesas hubo una mayor prevalencia de depresión, y que mayores niveles de ansiedad y depresión se asociaron con una menor eficacia percibida para realizar acciones para bajar de peso. Entre mayor fue la ansiedad, menor fue la disminución del peso corporal en las pacientes.
\end{abstract}

Palabras clave: Obesidad; Nutrición; Ansiedad; Depresión; Sobrepeso; Autoeficacia.

\begin{abstract}
In order to explore the relationship between depression, anxiety, self-efficacy for weight loss and body weight change in overweight and obese women undergoing nutritional treatment, the IDARE, the BDI-II and the Self-efficacy scales, were applied to 122 patients in two "family medicine" clinics in Mexico City. Results revealed higher prevalence of depression among obese participants, and higher levels of anxiety and depression associated with lower perceived efficacy for performing actions that promote weight loss. Higher anxiety levels related to a less weight reduction in the patients.
\end{abstract}

Key words: Obesity; Nutrition; Anxiety; Depression; Overweigh; Self-efficacy.

\section{INTRODUCCIÓN}

$\mathrm{E}$ 1 sobrepeso y la obesidad han aumentado en todas las edades, regiones y grupos socioeconómicos (James, 2004; Stein y Colditz, 2004; Wang y Lobstein, 2006). Particularmente, México ocupa el segundo lugar en el mundo en cuanto a obesidad en adultos y el primer lugar en obesidad infantil. La Encuesta Nacional de Salud y Nutrición (Gutiérrez et al., 2012) reportó que 34.4\% de los niños de entre 5 y 11 años de edad, 35\% de los adolescentes y 71.2\% de los adultos sufrían sobrepeso u obesidad.

La Organización Mundial de la Salud (OMS) (2012) define el sobrepeso y la obesidad como una acumulación excesiva de grasa que puede ser perjudicial para la salud, cuya causa fundamental es un desequilibrio entre las calorías que se consumen y las que se gastan. Para identificar estos padecimientos se

\footnotetext{
${ }^{1}$ División de Investigación y Posgrado, Facultad de Psicología, Ciudad Universitaria, Universidad Nacional Autónoma de México, Av. Universidad 3004, Del. Coyoacán, Col. Copilco Universidad, 04510 Ciudad de México, México, tels. (55)56-23-70-76 y (55)54-15-65-60, correo electrónico: csilva@posgrado.unam.mx. Artículo recibido el 9 de abril y aceptado el 6 de junio de 2018.
} 
emplea el índice de masa corporal (IMC), que es un coeficiente del peso expresado en kilogramos, dividido entre el cuadrado de la altura en centímetros. Según la OMS (2012), se considera sobrepeso un IMC entre 25 y $29 \mathrm{~kg} / \mathrm{m}^{2}$, y obesidad un IMC superior a $30 \mathrm{~kg} / \mathrm{m}^{2}$.

$\mathrm{Al}$ aumentar el grado de obesidad en una persona, aumenta igualmente el riesgo de padecer enfermedades crónicas, como diabetes, hipertensión, enfermedades cardiovasculares y diversos tipos de cáncer (Stein y Colditz, 2004), padecimientos todos ellos que implican una mayor utilización de los servicios de salud y una disminución en la esperanza y calidad de vida de quienes las sufren (Hussain y Bloom, 2011).

Tanto el sobrepeso como la obesidad se han asociado también con alteraciones psicológicas. Diversos estudios han discutido la asociación entre la depresión y la obesidad. Por ejemplo, Fabricatore y Wadden (2004) y Wardle y Cooke (2005) reportan no haber encontrado diferencias significativas en los niveles de depresión entre personas obesas y con peso normal (normopeso); sin embargo, Roberts, Kaplan, Shema y Strawbridge (2000), Roberts, Deleger, Strawbridge y Kaplan (2003) y Cofré, Angulo y Riquelme (2014) hallaron importantes asociaciones entre un elevado nivel de depresión y el sobrepeso. A su vez, autores como Puhl y Brownell (2001) argumentan que las personas obesas enfrentan frecuentemente estereotipos, prejuicios o conductas discriminatorias por parte de quienes las rodean, lo que propicia tal sintomatología.

Según la OMS (2015), la depresión es un trastorno que se caracteriza por tristeza, pérdida de interés o placer, sentimientos de culpa, falta de autoestima, trastornos del sueño o del apetito, sensación de cansancio y falta de concentración, y puede llegar a ser crónica o recurrente y dificultar sensiblemente el desempeño en el trabajo o la escuela y la capacidad para afrontar la vida diaria. En su forma más grave, puede conducir al suicidio. $\mathrm{Si}$ es leve, se puede tratar sin necesidad de medicamentos, pero cuando tiene un carácter moderado o grave se puede recurrir a ellos o requerir psicoterapia profesional.

En un metaanálisis realizado por Luppino et al. (2010), en el cual se analizaron artículos longitu- dinales publicados entre 1997 y 2008, se observó una asociación entre la depresión y la obesidad, encontrándose que las personas obesas presentaban un riesgo de $55 \%$ de padecer depresión debido a su percepción de estar lejos del ideal corporal de delgadez, lo que sugiere que el nivel de depresión en las personas obesas no solo se explica por un IMC elevado, sino también por una elevada insatisfacción corporal (Werrij, Mulkens, Hospers y Jansen, 2006). Esta interacción de variables explicaría por qué las personas obesas que buscan tratamiento para bajar de peso muestran mayores índices de depresión que las personas obesas que no lo hacen (Scott, McGee, Wells y Oakley, 2008; Wardle y Cooke, 2005).

Adicionalmente, la comorbilidad de la obesidad con la depresión también se asocia con problemas de salud en general y, lo que es más grave, los pacientes obesos parecen ser más resistentes a los antidepresivos que los pacientes con peso normal, lo cual dificulta el tratamiento de ambas condiciones (Jantaratnotai, Kanokwan, Lee y McIntyre, 2016).

Otra forma de alteración psicológica que se halla frecuentemente relacionada con el sobrepeso y la obesidad es la sintomatología ansiosa. Diversas investigaciones (de Wit, van Straten, van Herzen, Penninx y Cuijpers, 2009; Roberts et al., 2000; Scott et al., 2008) han dado cuenta de una asociación positiva entre el IMC y la sintomatología de ansiedad, definida como una reacción emocional que el individuo no puede controlar y que representa una preocupación o inquietud excesivas acerca de situaciones que tienen resultados inciertos (Martínez, Fernández, Navarro y Martínez, 2010). La diferencia entre la ansiedad normal y la patológica es que esta última conduce a suponer amenazas irreales y es de larga duración e intensidad, por lo que se vuelve difícil de controlar (Sierra, Ortega y Zubeidat, 2003). De hecho, los trastornos por ansiedad son los más comunes entre los pacientes con obesidad (Duarte, Mendonça, Santob, Lotufo y Wanga, 2017).

Algunos estudios (de Wit et al., 2009; Manzoni et al., 2009) señalan que las personas obesas que padecen ansiedad o depresión muestran un mayor riesgo a subir de peso debido a un aumento del apetito (síntoma que frecuentemente se en- 
cuentra presente en los trastornos de ansiedad y del estado de ánimo), lo que las induce a aumentar la frecuencia de ingestas o a comer más.

Aunado a lo anterior, hay evidencia que apunta a que los niveles elevados de ansiedad y depresión parecen afectar el éxito del tratamiento para bajar de peso, lo que resulta en un problema para modificarlo (Faulconbridge et al., 2009). Una variable que parece predecir en buena medida el éxito de tal tratamiento es la autoeficacia para controlar el peso corporal, es decir, lo eficaz que se siente una persona para modificar su nivel de actividad, sus hábitos alimentarios y sus patrones de consumo, teniendo como objetivo la pérdida de peso (Bas y Donmez, 2009; Campos y Pérez, 2007; Olander et al., 2013; Shin et al., 2011). Así, en algunos estudios se ha explorado la relación entre ansiedad, depresión y autoeficacia. Faulconbridge et al. (2009) encontraron una relación entre los atracones frecuentes, la depresión elevada y la baja autoeficacia para controlar el peso en un grupo de mujeres con sobrepeso. De manera similar, Bas y Donmez (2009) mostraron una correlación negativa entre la ansiedad-rasgo y la autoeficacia para bajar de peso en personas obesas, así como entre la ansiedad-rasgo y la pérdida de peso corporal después de un tratamiento nutricional de veinte semanas. Se ha considerado que un manejo adecuado de los síntomas ansiosos puede facilitar una mayor adherencia a los tratamientos para reducir el peso (Scott et al., 2008).

Dado que parece haber una relación entre el IMC y algunas variables psicológicas, tales como la autoeficacia, y que las personas obsesas manifiestan niveles bajos de autorregulación porque no pueden demorar la gratificación inmediata del comer, es necesario que los investigadores y los profesionales de la salud incluyan y evalúen dichos factores en los tratamientos y programas preventivos de control de peso (Menéndez y Orts, 2017).

Por todo lo anterior, el objetivo de esta investigación fue explorar la relación entre la ansiedad, la depresión, la autoeficacia para bajar de peso y el cambio de peso corporal en un grupo de pacientes obesas sometidas a tratamiento nutricional, para lo cual se efectuó un estudio trasversal con un alcance correlacional.

\section{MÉTODO}

\section{Participantes}

Se llevó a cabo una selección intencional no probabilística, por medio de la cual se obtuvo la participación consentida e informada de 122 mujeres con sobrepeso u obesidad, cuyas edades fluctuaron entre 20 y 72 años $(M=44.77$, D.E. $=12.05)$. Todas ellas recibían un tratamiento nutricional para el control de peso, para lo cual asistían a dos clínicas de medicina familiar de la Ciudad de México. Ambas clínicas pertenecen al sistema público de salud y en ellas se atiende a pacientes con diversos padecimientos por tratarse de clínicas de primer contacto. Las pacientes incluidas en este estudio fueron aquellas que habían solicitado los servicios del servicio de nutrición ya que mostraban interés por bajar de peso. Fueron excluidas de la investigación quienes sufrían padecimientos que pudieran alterar su peso corporal, como diabetes o alteraciones de la glándula tiroides, así como quienes recibían tratamientos con antidepresivos o ansiolíticos o estaban embarazadas.

Del total de participantes, la mitad de ellas presentaban sobrepeso al inicio del tratamiento (IMC 25.63-29.94; $\mathrm{M}=27.94$, D.E. $=1.11$ ) y la otra mitad obesidad (IMC 32.51-56.60; $\mathrm{M}=37.37$, D.E. $=4.84$. En la Tabla 1 se presenta la distribución de frecuencias de los distintos niveles de ansiedad y depresión en las participantes.

Tabla 1. Niveles de ansiedad y depresión por categoría de peso corporal.

\begin{tabular}{|c|l|r|r|r|c|}
\hline \multirow{3}{*}{ Variable } & \multirow{2}{*}{ Nivel } & \multicolumn{2}{|c|}{ Sobrepeso } & \multicolumn{2}{c|}{ Obesidad } \\
\cline { 3 - 6 } & & Total & $\mathbf{\%}$ & Total & $\mathbf{\%}$ \\
\hline \multirow{4}{*}{ Ansiedad } & Mínima & 12 & 23.5 & 10 & 19.6 \\
\cline { 2 - 6 } & Leve & 17 & 33.3 & 10 & 19.6 \\
\cline { 2 - 6 } & Moderada & 11 & 21.6 & 12 & 23.5 \\
\cline { 2 - 6 } & Severa & 11 & 21.6 & 19 & 37.3 \\
\hline \multirow{5}{*}{ Depresión } & Mínima & 38 & 74.5 & 26 & 51.0 \\
\cline { 2 - 6 } & Leve & 8 & 15.7 & 7 & 13.7 \\
\cline { 2 - 6 } & Moderada & 3 & 5.9 & 10 & 19.6 \\
\cline { 2 - 6 } & Severa & 2 & 3.9 & 8 & 15.7 \\
\hline
\end{tabular}




\section{Aparatos}

Para medir y pesar a las participantes se utilizaron las básculas con las que se trabajaba en cada una de las clínicas; así, en la primera se usó una báscula digital con estadímetro y cálculo automático de IMC con capacidad para $250 \mathrm{k}$, y en la segunda una báscula con estadímetro con capacidad para $140 \mathrm{k}$.

\section{Instrumentos}

Inventario de Ansiedad Rasgo-Estado (IDARE) (Spielberger y Díaz-Guerrero, 1975).

Es un inventario autoaplicable, estandarizado para México por Spielberger y Díaz-Guerrero (1975), el cual evalúa dos conceptos de ansiedad: la ansiedad como estado (condición emocional transitoria) y la ansiedad como rasgo (propensión a la ansiedad relativamente estable). En la presente investigación únicamente se empleó la escala de ansiedad-rasgo debido a que la presencia prolongada de ansiedad y depresión aumenta hasta en $70 \%$ la probabilidad de subir de peso y disminuye la probabilidad de perderlo (de Wit, van Straten, Famers, Cuijpers y Pennix, 2015). Este instrumento consta de veinte ítems cuyas opciones de respuesta son "Casi nunca", "Algunas veces", "Frecuentemente" y "Casi siempre", con las cuales se obtiene una puntuación total que puede ir de 20 a 80. La confiabilidad test-retest (estabilidad) de la escala A-Rasgo es relativamente alta: entre .73 y .86; asimismo, la consistencia interna oscila entre .89 y .90 . En este estudio la consistencia interna, medida por el coeficiente alfa de Cronbach $(\alpha)$, fue de .91. Dado que el IDARE no cuenta con puntos de corte, éstos se determinaron mediante cuartiles, considerándose como ansiedad mínima un puntaje entre 20 y 31 puntos, leve entre 32 a 40 puntos, moderada entre 41 a 50 puntos, y grave un puntaje de 51 puntos o más.

\section{Inventario de Depresión de Beck (BDI-II)} (Beck, Ward, Mendelson, Mock y Erbaugh, 1961). Es un instrumento de autoinforme compuesto por veintiún reactivos cuya finalidad es tamizar el nivel de depresión. Se emplea para diferenciar rápidamente los posibles casos de depresión en adultos y adolescentes mayores de 13 años de edad.
Cada reactivo se evalúa mediante una escala de cuatro puntos, que van de 0 a 3 , donde 0 es la ausencia del síntoma y 3 su intensidad más elevada. La puntuación final se determina mediante la suma de las elecciones en los ítems. Los niveles de depresión que se obtienen mediante el BDI-II son, a saber: mínimo, 0 a 13 puntos; leve, 14 a 19 puntos; moderado, 20 a 28 puntos, y severo, 29 a 63 puntos. En su validación mexicana (Jurado et al., 1998), se obtuvo una consistencia interna de .92 en pacientes psiquiátricos de consulta externa, y de .93 en estudiantes universitarios. La consistencia interna obtenida en el presente estudio fue de .90 .

\section{Escala de Autoeficacia para Bajar de Peso}

(Campos y Pérez, 2007).

Creada para identificar la eficacia percibida en pacientes mujeres, el instrumento está constituido por 38 reactivos, los cuales se dividen entre las tres dimensiones que evalúa: regulación de la compra de alimentos (diez reactivos); control de los hábitos alimentarios o ingesta (dieta) (quince reactivos), y adopción y mantenimiento de la actividad física (trece reactivos). Utiliza una escala tipo Likert de 7 puntos, que van de "No estoy segura de poder hacerlo", hasta "Completamente segura de poder hacerlo". Se considera un nivel alto de autoeficacia cuando se obtiene en la escala total una sumatoria de entre 190 y 228 puntos. En cuanto a las subescalas, hay un nivel alto cuando los puntajes se encuentran entre 50 y 60 puntos en la subescala de compra de alimentos, entre 75 y 90 en la de dieta y entre 65 y 78 puntos en la de ejercicio. Asimismo, se considera un nivel bajo de autoeficacia cuando se obtiene en la escala total una sumatoria de entre 0 y 189 puntos. Respecto a las subescalas, hay un nivel bajo cuando los puntajes van de 0 a 49 puntos en la subescala de compra de alimentos, de 0 a 74 en la de dieta y de 0 a 64 en la de ejercicio. Al momento de su elaboración con una muestra de 170 mujeres chilenas, se obtuvo una consistencia interna que osciló entre .93 y .96 en la escala total y en cada una de las subescalas. En el presente estudio la consistencia interna obtenida fue de .97 en la escala completa, de .94 en la subescala de control de los hábitos alimentarios, de .95 en la de adopción y mantenimiento de la actividad física, y de .92 en la de regulación en la compra de alimentos. 


\section{Procedimiento}

Para realizar este estudio, se solicitó el apoyo institucional de dos clínicas de medicina familiar del sistema público de salud. Una vez aprobado el desarrollo de la investigación, se explicó al personal y a las pacientes el objetivo del estudio, y tras conseguir su firma del consentimiento informado, se recabaron los datos de interés. Las aplicaciones de los instrumentos se efectuaron de manera individual en los consultorios de los centros de salud y fueron hechas por psicólogos y nutriólogos expertos.

En un primer momento, se elaboró la historia clínica de las pacientes que acudían al servicio de nutrición. El formato de la historia clínica es el mismo que se utiliza en las clínicas cuando los pacientes asisten a consulta por primera vez, e incluye antecedentes heredo-familiares, historia de enfermedades o cirugías previos al momento de la consulta, el motivo de la misma y algunos datos sociodemográficos, como el nivel educativo, el número de hijos, la situación laboral y otros.

A cada paciente se le asignó una dieta de acuerdo a sus necesidades y a los hábitos que cada una de ellas reportó. En todos los casos, se consideraron dietas con una disminución calórica de $20 \%$ respecto a la ingesta reportada. En el caso de que cumplieran con los criterios de inclusión, se les invitaba a participar en el estudio. Antes de comenzar la evaluación se les brindó información sobre los objetivos, los riesgos y los beneficios del estudio; también se hizo hincapié en que su participación era totalmente voluntaria y estrictamente confidencial, pues la información sería utilizada únicamente con fines exclusivamente estadísticos. Si las participantes aceptaban participar, se procedía a la aplicación de los instrumentos psicológicos, lo que se hizo únicamente en la primera consulta. Finalmente, se les citó en cuatro ocasiones, con un intervalo de un mes entre cada consulta, para el seguimiento del tratamiento nutricional. En cada consulta se registró su peso corporal.

Se calculó el IMC de las participantes y se les dividió por categorías de peso corporal (sobrepeso y obesidad), según los criterios propuestos por la OMS.

\section{RESULTADOS}

Al comparar mediante pruebas $t$ los puntajes de ansiedad obtenidos por las pacientes con sobrepeso y aquellas con obesidad, no se encontraron diferencias estadísticamente significativas $(t[100]$ $=-.979, p>.05$ ); en cambio, se encontraron diferencias en los puntajes de depresión $(t[100]=-2.871$, $p<.01$ ), siendo mayor el grado de depresión en las pacientes con obesidad $(\mathrm{M}=15.7$, D.E. $=11.0)$, en comparación con las pacientes con sobrepeso $(\mathrm{M}=10.3$; D.E. $=7.8)$. La correlación entre los puntajes de ansiedad y depresión fue de $r=.77$, $p<.001$.

Considerando el puntaje total del instrumento, se observó una autoeficacia escasa para bajar de peso en $69.6 \%$ de las participantes. Al analizar cada una de las subescalas, se encontró que la mayoría de ellas se percibían como poco eficaces para controlar sus hábitos alimentarios $(77.5 \%)$ y para adoptar y mantener actividades físicas (69.6\%); $50 \%$ se percibían como poco eficaces para regular la compra de sus alimentos. No se encontraron diferencias estadísticamente significativas entre las pacientes con sobrepeso y con obesidad en ninguna de las subescalas de la Escala de Autoeficacia para Bajar de Peso ni en el puntaje total.

En la Tabla 2 pueden apreciarse las medias y las desviaciones estándar en los puntajes de cada una de las variables.

Tabla 2. Medias de ansiedad, autoeficacia y depresión por categoría de peso corporal.

\begin{tabular}{|l|c|c|c|c|}
\hline \multirow{2}{*}{\multicolumn{1}{c|}{ Variable }} & \multicolumn{2}{c|}{ Sobrepeso } & \multicolumn{2}{c|}{ Obesidad } \\
\cline { 2 - 5 } & M & D.E. & M & D.E. \\
\hline Ansiedad & 34.75 & 2.77 & 35.25 & 1.11 \\
\hline Compra de alimentos & 55.12 & 3.09 & 58.37 & 3.25 \\
\hline Dieta & 79.87 & 5.91 & 86.00 & 4.60 \\
\hline Ejercicio & 73.62 & 7.58 & 79.12 & 6.11 \\
\hline Depresión & 13.37 & 2.49 & 18.37 & 3.46 \\
\hline
\end{tabular}

Hecho lo anterior, se obtuvieron correlaciones de Pearson entre los puntajes de autoeficacia para bajar de peso, ansiedad y depresión. Se hallaron correlaciones significativas superiores a .60 entre las tres subescalas de autoeficacia para bajar de peso (Tabla 3). 
Tabla 3. Correlación entre los factores de autoeficacia para bajar de peso, ansiedad y depresión.

\begin{tabular}{|l|c|c|c|c|c|}
\hline \multicolumn{1}{|c|}{ Variables } & $\mathbf{2}$ & $\mathbf{3}$ & $\mathbf{4}$ & $\mathbf{5}$ & $\mathbf{6}$ \\
\hline 1. Control de ingesta & $.64 * *$ & $.74 * *$ & $.91 * *$ & $-.30 * *$ & $-.22 *$ \\
\hline 2. Actividad física & & $.62 * *$ & $.87 * *$ & $-.25 *$ & -.17 \\
\hline 3. Compra de alimentos & & & $.86 * *$ & $-.25 * *$ & $-.22 *$ \\
\hline 4. Total de autoeficacia & & & & $-.31 * *$ & $-.23 *$ \\
\hline 5. Ansiedad & & & & & $.77 * *$ \\
\hline 6. Depresión & & & & & \\
${ }^{*} p<.05 ; * p<.01$ & & & & \\
\end{tabular}

Asimismo, se encontró que entre mayores eran los niveles de ansiedad, menor fue la autoeficacia para bajar de peso y para llevar a cabo cada una de las actividades que ayudarían a lograr dicho fin, tales como controlar la ingesta de alimentos, iniciar y mantener la actividad física y regular la compra de alimentos. En cuanto a su relación con la depresión, se encontró que a mayor depresión, menor fue la autoeficacia para controlar la ingesta de alimentos y para regular su compra. En cambio, no se halló relación entre la depresión y la autoeficacia para realizar actividad física.

Se realizó una correlación parcial para conocer si, controlando estadísticamente el número de consultas al que asistieron las pacientes, había relación entre el cambio de peso corporal y la autoeficacia, la ansiedad y la depresión. El cambio de peso corporal se calculó restando el peso alcanzado en la última consulta del peso con el que las participantes habían llegado a la primera consulta. Cabe señalar que no todas las pacientes asistieron a las cuatro consultas y que solo 42 asistieron a tres de ellas. Únicamente se encontró una correlación significativa entre la ansiedad y el cambio de peso corporal $(r=-.202, p<.05)$; esto es, a mayor ansiedad, menor cambio del peso corporal, con independencia del número de asistencias a la consulta nutricional.

\section{DISCUSIÓN}

El presente estudio se realizó con la finalidad de explorar la relación entre depresión, ansiedad, autoeficacia para bajar de peso y cambio de peso corporal en pacientes con sobrepeso y obesidad sometidas a tratamiento nutricional, por lo que se evaluó a 122 pacientes a quienes se les aplicaron el IDARE, el BDI-II y la Escala de Autoeficacia para Bajar de Peso.

Los resultados indican que las pacientes sometidas al tratamiento de reducción de peso eran aquellas con mayor IMC y con mayores niveles de depresión, lo que coincide con lo reportado en otras investigaciones que han documentado esa misma relación en pacientes que buscan programas de reducción de peso (Bjerkeset, Romundstad, Evans y Gunnell, 2008; Gadalla, 2009; Rivenes, Harvey y Mykletunc, 2009; Wardle y Cooke, 2005; Werrij et al., 2006). El hecho de que las personas obesas muestren un mayor nivel de depresión que las personas con sobrepeso hace pensar en la posibilidad de que las dificultades que frecuentemente enfrentan, tanto de tipo físico como social (Puhl y Heuer, 2009; World Health Organization, 2000), constituyan una importante causa de dicha depresión; sin embargo, no es posible asumir que los problemas emocionales sean una consecuencia inevitable de la obesidad (Luppino et al., 2010; Wardle y Cooke, 2005) -por lo que será necesario el desarrollo de más líneas de investigación que aclaren tal relación-, ni que las intervenciones para el control de peso deban incluir el manejo de trastornos afectivos o del estado de ánimo (Romain, Marleau y Baillot, 2018).

En lo que refiere a la ansiedad, no se encontraron diferencias estadísticamente significativas entre las pacientes con sobrepeso y con obesidad. Sin embargo, considerando el total de las participantes, poco más de la mitad de ellas sufrían ansiedad en un grado moderado o severo, por lo que parece importante detectar y tratar dicha sintomatología de manera específica, pues en otros estudios se ha observado que el manejo de la ansiedad en pacientes que buscan tratamiento para bajar de peso favorece la adherencia al tratamiento nutri- 
cional y la disminución de la frecuencia o la cantidad de ingesta de alimentos (de Wit et al., 2009; Manzoni et al., 2009; Scott et al., 2008).

En general, se observó una baja autoeficacia para perder peso en la mayoría de las pacientes; casi $70 \%$ de ellas se percibían como poco eficaces para modificar sus hábitos de ingesta de alimentos y para iniciar y mantener una actividad física, lo que resulta ser un indicador negativo, pues si no se modifica el balance energético para que éste sea mayor al consumo, no se producirá la reducción de peso corporal (Cordero et al., 2014). Al mismo tiempo, las participantes reportaron sentirse menos eficaces para emprender dichas actividades cuando experimentaban emociones negativas, no disponían de tiempo para ello u otras personas interferían (por ejemplo, comer por el mero hecho de ver a otras personas comiendo). Coincidiendo con los resultados de otras investigaciones, también se observó que entre mayor era la ansiedad y la depresión, menor era la autoeficacia percibida para llevar a cabo acciones que favorecieran la reducción del peso corporal (Bas y Donmez, 2009; Faulconbridge et al., 2009), y asimismo que a mayor ansiedad, menor la disminución del peso corporal de las pacientes (Scott et al., 2008).

Dado lo anterior, se sugiere que en el tratamiento nutricional para la reducción de peso dirigido a personas con sobrepeso y obesidad se evalúen no sólo sus requerimientos fisiológicos para diseñar una propuesta nutricional, sino también las variables psicológicas que pueden obstaculizar la disminución del peso. Es probable que la causa del malestar psicológico sea el peso corporal, pero ha de identificarse en cada caso individualizando la causa específica del malestar emocional.

Es necesario integrar en los protocolos de intervención nutricional el manejo psicológico o psiquiátrico de los pacientes, lo que podría elevar los buenos resultados del tratamiento en virtud de que se sabe que cuando surgen episodios de tristeza profunda o ansiedad, o de emociones negativas en general, se eleva la propensión a consumir grandes cantidades de comida, sobre todo carbohidratos (Acevedo y Gómez-Peresmitré, 2013; Geliebter y Aversa, 2003), lo que no ocurre en personas con normopeso (Ramos, González y Silva, 2016).

Entre las limitaciones de este trabajo debe decirse que, debido a que la población de las clínicas en las que se trabajó es fluctuante y que los recursos de que disponen obligan a que las intervenciones sean breves, en este estudio se empleó un diseño de corte trasversal, lo que solamente permitió obtener un pequeño segmento de información. Para alcanzar una mejor comprensión de este fenómeno es necesario utilizar un diseño longitudinal durante aproximadamente un año (Laquatra, 2004), tiempo en el que, según la literatura aquí revisada, se considera adecuada una disminución de $15 \%$ del peso corporal. Se podrán así hacer varios cortes con evaluaciones que hagan posible conocer las fluctuaciones e interacciones de las variables de ansiedad, depresión y autoeficacia para bajar de peso a lo largo del proceso.

\section{REFERENCIAS}

Acevedo R., S.P. y Gómez-Peresmitré, G. (2013). Propiedades psicométricas de la Escala de Factores de Riesgo Asociados con Trastornos de la Alimentación (EFRATA-II) en preadolescentes mexicanos. Psicología y Salud, 23(2), $251-259$.

Bas, M. y Donmez, S. (2009). Self-efficacy and restrained eating in relation to weight loss among overweight men and women in Turkey. Appetite, 52(1), 209-216. doi: 10.1016/j.appet.2008.09.017.

Beck, A.T., Ward, C.H., Mendelson, M., Mock, J. y Erbaugh, J. (1961). An inventory for measuring depression. Archives of General Psychiatry, 4, 561-571.

Bjerkeset, O., Romundstad, P., Evans, J. y Gunnell, D. (2008). Association of adult body mass index and height with anxiety, depression, and suicide in the general population: The HUNT Study. American Journal of Epidemiology, 167(2), $193-202$. doi: 10.1093/aje/kwm280.

Campos R., S. y Pérez E., C. (2007). Autoeficacia y conflicto decisional frente a la disminución del peso corporal en mujeres. Revista Chilena de Nutrición, 34(3), 213-218.

Cofré, A., Angulo D., P. y Riquelme M., E. (2014). Ansiedad y depresión en pacientes obesos mórbidos: efectos a corto plazo de un programa orientado a la disminución de la sintomatología. Summa Psicológica, 11(1), 89-98.

Cordero M., A., Piñero A., O., Vilar N., M., García J., S., Verazaluce J., G., García I., G. y López A., S. (2014). Programas de actividad física para reducir sobrepeso y obesidad en niños y adolescentes; revisión sistemática. Nutrición Hospitalaria, 30(04), 727-740. doi: 10.3305/nh.2014.30.4.7680. 
de Wit, L.M., van Straten, A., van Herten, M., Penninx, B.W. y Cuijpers, P. (2009). Depression and body mass index, a u-shaped association. BioMed Central Public Health, 9. doi: 10.1186/1471-2458-9-14.

de Wit, L.M., van Straten, A., Lamers, B., Cuijpers, P. y Penninx, B.W. (2015). Depressive and anxiety disorders: Associated with losing or gaining weight over 2 years? Psychiatry Research, 227, 230-237. doi. 10.1016/j.psychres.2015.02.025 01651781/y 2015.

Duarte G., L., Mendonça C., B., Santo, M.A., Lotufo N., F. y Wanga Y., P. (2017). Morbidity persistence and comorbidity of mood, anxiety, and eating disorders among preoperative bariatric patients. Psychiatry Research, 257, 1-6.

Fabricatore, A.N. y Wadden, T.A. (2004). Psychological aspects of obesity. Clinics in Dermatology, 22(4), 332-337. doi: 10.1016/j.clindermatol.2004.01.006.

Faulconbridge, L.F., Wadden, T.A., Berkowitz, R.I., Sarwer, D.B., Womble, L.G, Hesson, L.A. y Fabricatore, A.N. (2009). Changes in symptoms of depression with weight loss: results of a randomized trial. Obesity, 17(5), 1009-1016. doi: 10.1038/ oby.2008.647.

Gadalla, T.M. (2009). Association of obesity with mood and anxiety disorders in the adult general population. Chronic Diseases in Canada, 30(1), 29-36.

Geliebter, A. y Aversa, A. (2003). Emotional eating in overweight, normal weight, and underweight individuals. Eating Behaviors, 3(4), 341-347. doi: 10.1016/S1471-0153(02)00100-9.

Gutiérrez J., P., Rivera D., J., Shamah L., T., Villalpando H., S., Franco, A., Cuevas N., L. y Hernández Á., M. (2012). Encuesta Nacional de Salud y Nutrición 2012. Resultados nacionales. Cuernavaca (México): Instituto Nacional de Salud Pública.

Hussain, S.S. y Bloom, S.R. (2011). The pharmacological treatment management of obesity. Postgraduate Medicine, 123(1), 34-44.

James, P.T. (2004). Obesity: The worldwide epidemic. Clinics in Dermatology, 22(4), 276-280. doi: 10.1016/j.clindermatol.2004.01.010

Jantaratnotai, N., Kanokwan, M., Lee, Y. y McIntyre, R.S. (2016). The interface of depression and obesity. Obesity Research \& Clinical Practice, 11, 1-10.

Jurado, S., Villegas, M.E., Méndez, L., Rodríguez, F., Loperena, V. y Varela, R. (1998). La estandarización del Inventario de Depresión de Beck para los residentes de la Ciudad de México. Salud Mental, 21(3), 26-31.

Laquatra, I. (2004). Nutrition for weight management. En L. K. Mahan y S. Escott-Stump (Eds.): Krause's food nutrition and diet therapy (11 ${ }^{\text {th }}$ ed.) (pp. 488-493). Philadelphia, PA: W.B. Saunders Co.

Luppino, F.S., de Wit, L.M., Bouvy, P.F., Stinjnen, T., Cuijpers, P., Penninx, B.W. y Zitman, F.G. (2010). Overweight, obesity, and depression: A systematic review and meta-analysis of longitudinal studies. Archives of General Psychiatry, 67(3), 220-229. doi: 10.1001/archgenpsychiatry.2010.2.

Manzoni, G.M., Pagnini, F., Gorini, A., Preziosa, A., Castelnuovo, G., Molinari, E. y Riva, G. (2009). Can relaxation training reduce emotional eating in women with obesity? An exploratory study with 3 months of follow-up. Journal of the American Dietetic Association, 109(8), 1427-1432. doi: 10.1016/j.jada.2009.05.004.

Martínez A., L., Fernández, C., Navarro I., G. y Martínez M., O. (2010). Trastornos de ansiedad en niños y adolescentes. En R. E. Ulloa, C. Fernández, H. D. Gómez, J. Ramírez y J. C. Reséndiz (Eds.): Guías clínicas del Hospital Psiquiátrico Infantil "Dr. Juan N. Navarro" (pp. 113-149). México: PSICOFARMA.

Menéndez G., L. y Orts C., M.I. (2017). Factores psicosociales y conductuales en la regulación del peso: autorregulación, autoeficacia y locus de control. Obesity Research \& Clinical Practice, 11, 1-10.

Olander, E.K., Fletcher, H., Williams, S., Atkinson, L., Turner, A. y French, D.P. (2013). What are the most effective techniques in changing obese individuals' physical activity self-efficacy and behavior: A systematic review and meta-analysis. International Journal of Behavioral Nutrition and Physical Activity, 10(29), doi: 10.1186/1479-5868-10-29.

Organización Mundial de la Salud (2012). Obesidad y sobrepeso. Nota descriptiva $N^{\circ} 311$. Ginebra: OMS. Disponible en http:// www.who.int/mediacentre/factsheets/fs3/es/index.html.

Organización Mundial de la Salud (2015). Temas de salud. Depresión. Ginebra: OMS. Disponible en http://www.who.int/topics/ depression/es/.

Puhl, R. y Brownell, K.D. (2001). Bias, discrimination, and obesity. Obesity Research, 9(12), 788-805. doi: 10.1038/oby.2001.108.

Pull, R.M. y Heuer, C.A. (2009). The stigma of obesity: A review and update. Obesity, 17(5), 941-964.

Ramos R., J.H., González A., K.E. y Silva, C. (2016). Efecto de la interacción entre el sexo y el peso sobre el comer emocional en adolescentes. Psicología y Salud, 26(1), 63-68.

Rivenes, A.C., Harvey, S.B. y Mykletun, A. (2009). The relationship between abdominal fat, obesity, and common mental disorders: results from the HUNT study. Journal of Psychosomatic Research, 66(4), 269-275. doi: 10.1016/j.jpsychores.2008.07.012.

Roberts, R.E., Deleger, S., Strawbridge, W.J. y Kaplan, G.A. (2003). Prospective association between obesity and depression: evidence from the Alameda County Study. International Journal of Obesity, 27(4), 514-521. doi: doi.10.1038/sj.ijo.0802204.

Roberts, R.E., Kaplan, G.A., Shema, S.J. y Strawbridge, W.J. (2000). Are the obese at greater risk for depression? American Journal of Epidemiology, 152(2), 163-170. doi: doi.10.1093/aje/152.2.163. 
Romain, A.J., Marleau, J. y Baillot, A. (2018). Impact of obesity and mood disorders on physical comorbidities, psychological well-being, health behaviours and use of health services. Journal of Affective Disorders, 225, 381-388. doi: doi.10.1016/j. jad.2017.08.065.

Scott, K.M., McGee, M.A., Wells, E. y Oakley, M.A. (2008). Obesity and mental disorders in the adult general population. Journal of Psychosomatic Research, 64(1), 97-105. doi: doi:10.1016/j.jpsychores.2007.09.006.

Shin, H., Shin, J., Liu, P.Y., Dutton, G.R., Abood, D.A. e Ilich, J.Z. (2011). Self-efficacy improves weight loss in overweight/ obese postmenopausal women during a 6-month weight loss intervention. Nutrition Research Journal, 31 (11), 822-828. doi: 10.1016/j.nutres.2011.09.022.

Sierra, J.C., Ortega, V. y Zubeidat, I. (2003). Ansiedad, angustia y estrés: tres conceptos a diferenciar. Revista Malestar e Subjetividade, 3(1), 10-59.

Spielberger, C.D. y Díaz-Guerrero, R.I. (1975). Inventario de Ansiedad: Rasgo-Estado. México: El Manual Moderno.

Stein, C.J. y Colditz, G.A. (2004). The epidemic of obesity. The Journal of Clinical Endocrinology \& Metabolism, 89(6), 25222525. doi: doi:10.1210/jc.2004-0288.

Wang, Y. y Lobstein, T.I.M. (2006). Worldwide trends in childhood overweight and obesity. International Journal of Pediatric Obesity, 1(1), 11-25. doi: 10.1080/17477160600586747.

Wardle, J. y Cooke, L. (2005). The impact of obesity on psychological well-being. Best Practice \& Research Clinical Endocrinology \& Metabolism, 19(3), 421-440. doi: 10.1016/j.beem.2005.04.006.

Werrij, M.Q., Mulkens, S., Hospers, H.J. y Jansen, A. (2006). Overweight and obesity: The significance of a depressed mood. Patient Education and Counseling, 62(1), 126-131. doi: 10.1016/j.pec.2005.06.016.

World Health Organization (2000). Obesity: Preventing and managing the global epidemic. Report of a WHO Consultation, WHO Technical Report Series 894. Geneve: WHO. 